\title{
Optimization For Supply Chain Models With Policies *
}

\author{
M. Herty ${ }^{\dagger}$ and C. Ringhofer ${ }^{\ddagger}$ \\ December 7, 2006
}

\begin{abstract}
We develop a methodology to investigate optimal dynamic policies for a large class of supply networks. The basic underlying model is a fluid dynamic model for continuous product flows in continuous time. Discrete as well as continuous controls are implemented.

KEYWORDS: Policy models, production lines, optimization

AMS CLASSIFICATION: 65N35, 65N05
\end{abstract}

\section{Introduction}

This papers is based on a fluid dynamic model for a supply chain, originally developed in [6]. Each individual supplier is modeled by a partial differential equation, expressing the conservation of product, a limited capacity of the supplier and the time delayed response of outputs to inputs. In essence, this model relates the principles involved in the fluid dynamic modeling of traffic flow $[18,21]$ to the supply network problem. In [6] this model has been rigorously derived from an automaton, corresponding to a deterministic discrete event simulation $[7,9,1]$ with few rather basic assumptions. These

*This work was supported by NSF grant DMS-0604986 and the Kaiserslautern Excellence Cluster 'Dependable adaptive systems and mathematical modeling'.

†TU Kaiserslautern, Department of Mathematics, PO Box 3049, D-67653 Kaiserslautern, Germany (herty@mathematik.uni-kl.de)

$\ddagger$ Department of Mathematics, Arizona State University, Tempe, AZ 85287-1804, USA (ringhofer@asu.edu). 
basic model has been extended in various ways, e.g., [3, 5, 14, 13, 19]. Here, we extend the known models to a dynamic network of processors and input buffers including machine priorities for parts. We are interested in optimization problems to determine time-dependent optimal policies for processing priorities of each processor. These models are relevant in particular for reentrant supply chains where parts have to pass through the same processor more than once. We assume that on each of the passes the processor can decide which part receives higher priority and will therefore be processed first. Based on partial differential equation we ask whether there is an optimal choice for deciding on the processing order. Depending on the nature of these decision this leads either to a mixed-integer programming model or to a optimal control problem with partial differential equations as constraints. We investigate both approaches and derive for the latter an adjoint calculus. We give numerical examples of simulation and optimization of the proposed policy model.

This paper is organized as follows. In Section 2 we develop the network model, based on minor modifications to the basic model from [6] for the model of a single node. Sections 3 and 4 are devoted to the policy optimization algorithm. Policies can be controlled either by discrete (in Section 4) or continuous (Section 3) control variables. Section 5 is devoted to numerical experiments for a small network, previously examined in $[10,17]$, which requires however a rather sophisticated decision making process.

\section{The Model}

\subsection{The model for a single processor with a buffer}

Each of the processors and buffers is described by an initial - boundary value problem for a hyperbolic conservation law, coupled to an ordinary differential equation. They are of the form

$$
\begin{gathered}
\text { (a) } \partial_{t} \rho+v \partial_{x} \rho=0, \quad(b) v \rho(0, t)=f(q),(c) \rho(x, 0)=\rho^{I}(x), \\
\partial_{t} q+f(q)-\lambda=0, \quad q(0)=q^{I} .
\end{gathered}
$$

$\rho(x, t)$ in (1) denotes the density of parts per stage as a function of time. The stage $x \in[0,1]$ is a virtual variable describing the degree of completion of the part. So a part enters at stage $x=0$ and leaves at stage $x=1$ after 
a given time $T=\frac{1}{v}$, the throughput time of the processor. The total work in progress (WIP) of the processor at any given time is therefore computed as $W(t)=\int_{0}^{1} \rho(x, t) d x$. We assume that initially there is a certain WIP distribution $\rho^{I}$ is given. The function $q(t)$ denotes the length of the buffer in front of the processor. The buffer feeds into the processor through the boundary condition (1)(b). The evolution of the buffer contents is governed by (2), where $\lambda(t)$ denotes the external influx into the buffer. The function $f(q)$ in $(2)$ governs the the flux from the buffer into the processor. It is of the form

$$
f(q)=\min \left\{c, \frac{q}{\varepsilon}\right\},
$$

where $c$ denotes the capacity of the processor, i.e. the processor can take on $c d t$ parts per infinitesimal time $d t$. $\varepsilon$ is a small parameter. This model has been used in [13] to simulate production flows on networks. However, it requires some explanation. This model is based on a rigorous model, derived in [6] as the large time continuum limit of a discrete automaton. This model is of the form

$$
\partial_{t} \rho+\partial_{x} f=0, \quad f=\min \{c, v \rho\}, \quad f(0, t)=\lambda(t)
$$

where the variables $\rho, c, v$ have the same meaning as in (1), and $\lambda(t)$ denotes the external influx. An upwind discretization of (3) by a method of lines approach would yield the system of ordinary differential equations

$$
\frac{d}{d t} \rho_{j}+\frac{1}{\Delta x}\left(f_{j}-f_{j-1}\right)=0, \quad f_{j}=\min \left\{c, v \rho_{j}\right\}, j=1, . ., J, \quad f_{0}=\lambda,
$$

Where $\Delta x \rho_{j}$ is the content of cell number $j$, located in $\left[x_{j-1}, x_{j}\right)$ and $\Delta x$ is the (uniform) spatial cell size. The connection between (3) and (1)-(2) is the following. Since the influx $f_{j-1}$ into cell $j$ is limited by $c$, a simple maximum principle argument implies that $v \rho \leq c$ will hold for all time - except in the first cell, where the influx $f_{0}$ is given by $\lambda(t)$. Thus we might as well drop the restriction on the flux in all subsequent cells, and the same solution is obtained by defining the cell fluxes by

$$
f_{j}=\left(\begin{array}{ccc}
\lambda & \text { for } & j=0 \\
\min \left\{c, v \rho_{1}\right\} & \text { for } & j=1 \\
v \rho_{j} & \text { for } & j=2, . ., J
\end{array}\right) .
$$

If the influx $\lambda$ exceeds the capacity $c$ this will result in a density $\rho_{1}$ of order $O\left(\frac{1}{\Delta x}\right)$, corresponding to a $\delta$ - function at $x=0$ in the continuous problem 
(3). These arguments have been made rigorous in [6]. Setting $q=\Delta x \rho_{1}$ and $\Delta x=\varepsilon$ yields the model (2) for the buffer length $q$. Replacing the discretized model in the cells $j=2, . ., J$ by their continuum limit again yields (1). So, (1)-(2) is - in this sense - equivalent to (3). It can also be interpreted as a smoothing out the discontinuous flux function

$$
f(q)=\left(\begin{array}{lll}
c & \text { for } & q \neq 0 \\
0 & \text { for } & q=0
\end{array}\right)
$$

and thereby avoiding the analytical problems, associated with (4).

Note that this trick greatly reduces the analytical complexity of the problem, since the conservation law (1) can be solved explicitly in terms of characteristics, giving $\rho(x, t)=\frac{1}{v} f\left(q\left(t-\frac{x}{v}\right)\right)$, and, in particular $v \rho(1, t)=f\left(t-\frac{1}{v}\right)$. So, the processor - outflux $v \rho(1, t)$ is just the buffer outflux $f$ delayed by the processing time $T=\frac{1}{v}$, and (1) could be replaced by an ordinary delay - differential equation with constant time delay. However, nothing is really gained by doing so, since the solution of the delay equation would require the storage of $f$ at previous time steps, whereas the solution of (1) requires the storage of $\rho(x, t)$ for the current time step, which amounts to the same.

\subsection{Networks}

We now consider a network of processors. So, we have $J$ processors, each modeled by (1) and (2). Modeling a network is easily achieved by linking influx and outflux via Kichhoff's law. So, we have

$$
\begin{gathered}
\text { (a) } \partial_{t} \rho_{j}+v_{j} \partial_{x} \rho_{j}=0, \quad\left(\text { b) } v_{j} \rho_{j}(0, t)=f\left(q_{j}\right), \quad(c) \rho_{j}(x, 0)=\rho_{j}^{I}(x),\right. \\
\text { (a) } \partial_{t} q_{j}+f\left(q_{j}\right)-\sum_{k=1}^{J} A_{j k} v_{k} \rho_{k}(1, t)-\lambda_{j}=0, \quad(b) q_{j}(0)=q_{j}^{I}, \quad j=1, . ., J .
\end{gathered}
$$

The matrix elements $A_{j k}$ denote the percentage of the output $v_{k} \rho_{k}(1, t)$ of processor $k$ going into the buffer $q_{j}$ of processor $j$. Because of mass conservation. the column sums $\sum_{j=1}^{J} A_{j k}$ will equal unity, except for the exit processors, which produce the final product of the network. $\lambda_{j}(t)$ in $(6)(\mathrm{a})$ are the external inputs into processor $j$. So, $\lambda_{j}=0$ will hold, except for the input processors, receiving external feeds. If every processor feeds all output into exactly one other processor, all the elements of the matrix $A$ will be either 0 or 1 , and $A^{T}$ is just the connectivity matrix of the graph describing the network. 


\subsection{Policies, multiple buffers and virtual processors}

We now consider the case when one processor has more than one buffer feeding into it. This is necessary for re-entrant networks, where one part has to go through the same processor more than once. So, this processor will have at least two buffers, for the parts going through it on, say, their first and second pass. This, in turn, requires a policy. Do we give priority of service to parts on their first pass (referred to as 'PUSH' policy) or there second pass (referred to as 'PULL' policy)? We model the situation of processor number $j$ being fed by $S(j)$ buffers, ordered in descending order by their priority, by replacing the processor number $j$ by $S(j)$ virtual processors with restricted capacities. They will, according to (5)-(6) satisfy the equations

$$
\begin{gathered}
\text { (a) } \partial_{t} \rho_{j s}+v_{j} \partial_{x} \rho_{j s}=0, \quad(b) v_{j} \rho_{j s}(0, t)=f_{j s}\left(q_{j s}\right),(c) \rho_{j s}(x, 0)=\rho_{j s}^{I}(x), \\
\text { (a) } \partial_{t} q_{j s}+f_{j s}\left(q_{j s}\right)-\sum_{k=1}^{J} \sum_{r=1}^{S(j)} A_{j s k r} v_{k} \rho_{k r}(1, t)-\lambda_{j s}=0, \quad(b) q_{j}(0)=q_{j}^{I}, \\
s=1, . ., S(j), j=1, . ., J
\end{gathered}
$$

The densities $\rho_{j s}, s=1, . ., S(j)$ now model $S(j)$ virtual copies of processor number $j$. We define the output fluxes of the queues by

$$
f_{1 j}=\min \left\{c_{j}, \frac{v_{j} q_{1 j}}{\varepsilon}\right\}, \quad f_{2 j}=\min \left\{c_{j}-f_{1 j}, \frac{v_{j} q_{2 j}}{\varepsilon}\right\} \ldots
$$

So, the highest priority buffer uses the capacity of the processor. The buffer with the second highest priority uses any left over capacity of the highest priority buffer, and so on. We write this in a more compact form by introducing the effective capacities $\mu_{j s}$ for the buffer $s$ in front of processor $j$, and defining

$$
f_{j s}=\min \left\{\mu_{j s}, \frac{v_{j} q_{j s}}{\varepsilon}\right\}, \quad \mu_{j s}=c_{j}-\sum_{r=1}^{s-1} f_{j r}, \quad s=1, . ., S(j) .
$$

To avoid having to use a tensor instead of the connectivity matrix $A$, we encode the above idea by simply making the buffer - to - processor flux function $f$ in (6) formally dependent on all buffers $q=\left(q_{1}, . ., q_{J}\right)$, giving
(a) $\partial_{t} \rho_{j}+v_{j} \partial_{x} \rho_{j}=0$
(b) $v_{j} \rho_{j}(0, t)=f_{j}(q)$,
(c) $\rho_{j}(x, 0)=\rho_{j}^{I}(x)$,
(a) $\partial_{t} q_{j}+f_{j}(q)-\sum_{k=1}^{J} A_{j k} v_{k} \rho_{k}(1, t)-\lambda_{j}=0, \quad(b) q_{j}(0)=q_{j}^{I}, \quad j=1, . ., J$. 


\subsection{A simple example}

To illustrate that the above setting can actually be applied to quite complex situations, we consider the following 'toy problem'. We assume a simple process involving two processors in sequence. So the path of a part would be in $\rightarrow P_{1} \rightarrow P_{2} \rightarrow$ out. However, the process only works $70 \%$ of the time and has to be repeated for $30 \%$ of the parts. We give it two try's, and discard the remaining $9 \%$ for which both try's have been unsuccessful. So, over all, we have the possible paths in $\rightarrow P_{1} \rightarrow P_{2} \rightarrow$ out for $70 \%$ of the parts, in $\rightarrow P_{1} \rightarrow P_{2} \rightarrow P_{1} \rightarrow P_{2} \rightarrow$ out for $30 \%$ of $70 \%=21 \%$ of the parts, and $i n \rightarrow P_{1} \rightarrow P_{2} \rightarrow P_{1} \rightarrow P_{2} \rightarrow$ trash for the remaining $30 \%$ of $30 \%=9 \%$ of the parts. Thus, each of the two processors has two buffers, for the first and second pass parts, in front of it, and has to be replaced by two virtual processors. The structure is schematically depicted in Figure 1

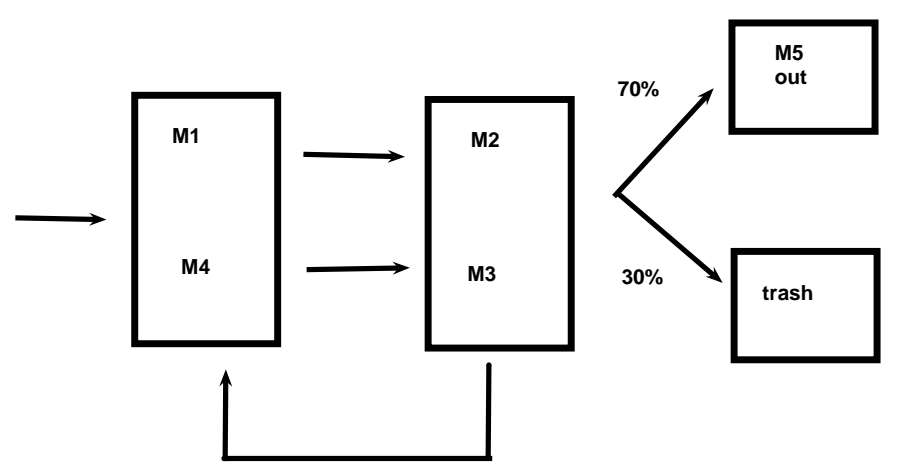

Figure 1: Two passes two processors $70 \%$ success rate.

Using a 'PUSH' policy, giving priority to the parts on their first path, we have the sequence in $\rightarrow M_{1} \rightarrow M_{3} \rightarrow M_{5}$ for $70 \%$ of the parts, in $\rightarrow M_{1} \rightarrow$ $M_{3} \rightarrow M_{2} \rightarrow M_{4} \rightarrow M_{5}$ for $21 \%$ of the parts and $i n \rightarrow M_{1} \rightarrow M_{3} \rightarrow M_{2} \rightarrow$ $M_{4} \rightarrow M_{6}$ for $9 \%$ of the parts. $M_{1}, M_{3}$ are the high priority copies of $P_{1}, P_{2}$ and $M_{2}, M_{4}$ are the low priority copies. Since we want to record the number of parts successfully processed and discarded, $M_{5}$ denotes the output and $M_{6}$ denotes the 'trash can'. This gives the $6 \times 6$ connectivity matrix $A$ and the 
influx vector $\lambda$ as

$$
A=\left(\begin{array}{cccccc}
0 & 0 & 0 & 0 & 0 & 0 \\
1 & 0 & 0 & 0 & 0 & 0 \\
0 & 0.3 & 0 & 0 & 0 & 0 \\
0 & 0 & 1 & 0 & 0 & 0 \\
0 & 0.7 & 0 & 0.7 & 0 & 0 \\
0 & 0 & 0 & 0.3 & 0 & 0
\end{array}\right), \quad \lambda=\left(\begin{array}{c}
\lambda_{1}(t) \\
0 \\
0 \\
0 \\
0 \\
0
\end{array}\right) .
$$

The flux functions in (11) would be given by

$$
\begin{gathered}
f_{1}=\min \left\{c_{1}, \frac{v_{1} q_{1}}{\varepsilon}\right\}, \quad f_{2}=\min \left\{c_{2}, \frac{v_{2} q_{2}}{\varepsilon}\right\}, \\
f_{3}=\min \left\{c_{1}-f_{1}, \frac{v_{1} q_{3}}{\varepsilon}\right\}, \quad f_{4}=\min \left\{c_{2}-f_{2}, \frac{v_{4} q_{4}}{\varepsilon}\right\}, \\
f_{5}=f_{6}=0 .
\end{gathered}
$$

Note, that the definition of $f$ is implicit, and therefore $f_{3}$ depends on $q_{1}$ as well as $q_{3}$ - hence the global dependence of the flux function $f$ on the whole buffer vector $q$. To change policy to a 'PULL' algorithm, giving priority to parts on their second pass, we would define the fluxes instead by

$$
\begin{gathered}
f_{1}=\min \left\{c_{1}-f_{3}, \frac{v_{1} q_{1}}{\varepsilon}\right\}, \quad f_{2}=\min \left\{c_{2}-f_{4}, \frac{v_{2} q_{2}}{\varepsilon}\right\}, \\
f_{3}=\min \left\{c_{1}, \frac{v_{1} q_{3}}{\varepsilon}\right\}, \quad f_{4}=\min \left\{c_{2}, \frac{v_{4} q_{4}}{\varepsilon}\right\} .
\end{gathered}
$$

\subsection{Network optimization}

The subject of the present paper is the optimization of the policies in the network. So, we do not choose a fixed set of rules for the flux functions and the routing matrix in (10)-(11), but let them depend on some control variables $u(t)=\left(u_{1}(t), . ., u_{N}(t)\right)$. So $(10)-(11)$ is replaced by

$$
\text { (a) } \partial_{t} \rho_{j}+v_{j} \partial_{x} \rho_{j}=0, \quad(b) v_{j} \rho_{j}(0, t)=f_{j}(q, u),(c) \rho_{j}(x, 0)=\rho_{j}^{I}(x),
$$

(a) $\partial_{t} q_{j}+f_{j}(q, u)-\sum_{k=1}^{J} A_{j k}(u) v_{k} \rho_{k}(1, t)-\lambda_{j}=0, \quad(b) q_{j}(0)=q_{j}^{I}, \quad j=1, . ., J$. 
We will choose the control variables $u$ to optimize a functional $G(\rho, q, u)$. This leaves the issue how to choose a dynamically changing policy, depending on the control variables $u(t)$. There are of course many different possibilities to do so. To give the reader some idea, we reconsider the example in Section 2.4. Instead of deciding a priori on a 'PUSH' or 'PULL' policy, defining the fluxes by (12) or (13), we build a convex combination of the two policies. We define

$$
\begin{gathered}
f_{1}=u_{1} f_{1}^{\text {push }}+\left(1-u_{1}\right) f_{1}^{\text {pull }}, \quad f_{3}=u_{1} f_{3}^{\text {push }}+\left(1-u_{1}\right) f_{3}^{\text {pull }} \\
f_{2}=u_{2} f_{2}^{\text {push }}+\left(1-u_{2}\right) f_{2}^{\text {pull }}, \quad f_{4}=u_{2} f_{4}^{\text {push }}+\left(1-u_{2}\right) f_{4}^{\text {pull }} \\
f_{1}^{\text {push }}=\min \left\{c_{1}, \frac{v_{1} q_{1}}{\varepsilon}\right\}, \quad f_{1}^{\text {pull }}=\min \left\{c_{1}-f_{3}^{\text {pull }}, \frac{v_{1} q_{1}}{\varepsilon}\right\}, \\
f_{2}^{\text {push }}=\min \left\{c_{2}, \frac{v_{2} q_{2}}{\varepsilon}\right\}, \quad f_{2}^{\text {pull }}=\min \left\{c_{2}-f_{4}^{\text {pull }}, \frac{v_{2} q_{2}}{\varepsilon}\right\}, \\
f_{3}^{\text {push }}=\min \left\{c_{1}-f_{1}^{\text {push }}, \frac{v_{1} q_{3}}{\varepsilon}\right\}, \quad f_{3}^{\text {pull }}=\min \left\{c_{1}, \frac{v_{1} q_{3}}{\varepsilon}\right\}, \\
f_{4}^{\text {push }}=\min \left\{c_{2}-f_{2}^{\text {push }}, \frac{v_{4} q_{4}}{\varepsilon}\right\}, \quad f_{4}^{\text {pull }}=\min \left\{c_{2}, \frac{v_{4} q_{4}}{\varepsilon}\right\} .
\end{gathered}
$$

Restricting the control variables $\left(u_{1}(t), u_{2}(t)\right)$ to the integers, $u(t) \in\{0,1\}^{2}$, means that we dynamically decide on a 'PUSH' or 'PULL' policy for each of the two processors. Numerically, restricting the controls $u$ to integers, leads to a mixed integer programming problem, for which there is limited convergence theory but excellent software [15], [8], c.f. section 4 for more details. In general, letting the control variables $u(t)$ vary continuously in $[0,1]^{N}$ leads to a more standard optimization problem and a more complex policy. Both approaches will be treated in Section 5. The convex combination of PULL and PUSH policies has to be interpreted stochastically. In time intervals of length $\frac{1}{c_{j}}$, when processor $j$ accepts a new part, the decision which buffer to serve is made randomly with probabilities $u_{j}$ and $1-u_{j}$, giving on average for large time scales, the mean fluxes in (16).

Note, that we also, in general, let the routing matrix $A$ in (15) depend on the control variables. This means that we include the case when we might dynamically change the path of a given part. 


\section{Continuous controls}

In this section we treat the case, when the control variables $u(t)$ are allowed to vary continuously, see also [14] for a discussion on a model without policies and virtual processors.

This yields a standard optimization problem, where the differential equations (14) and (15) are used as constraints on the functional $G$. Therefore the constrained optimization problem can be replaced by an unconstrained problem, using Lagrange multipliers. We denote the conservation laws for the $\rho_{j}$ in (14) by $C_{\rho}(\rho, q, u)=0$ and the ordinary differential equations (15) for the buffers by $C_{q}(\rho, q, u)=0$. Thus, we have to solve the constrained optimization problem

$$
G(\rho, q, u) \rightarrow \min , \quad C_{\rho}(\rho, q, u)=0, \quad C_{q}(\rho, q, u)=0
$$

for a given functional $G(\rho, q, u)$. Standard optimization theory implies that (17) can be replaced by minimizing the unconstrained functional

$$
G(\rho, q, u)+\left\langle\psi, C_{\rho}(\rho, q, u)\right\rangle+\left\langle\phi, C_{q}(\rho, q, u)\right\rangle \rightarrow \min
$$

over all possible functions $\rho, q, u, \psi, \phi$, where $\psi_{j}(x, t)$ is the Lagrange multiplier for $\rho_{j}$ in (14) and $\phi_{j}(t)$ is the Lagrange multiplier for $q_{j}$ in (15).

\subsection{Lagrange multipliers}

In order to use the Lagrange multiplier approach, we first have to re-formulate the constraints (14)-(15) in a weak form. We choose a fixed time horizon $T$ and define

$$
C_{\rho}(\rho, q, u)=0 \Longleftrightarrow\left\langle\psi, C_{\rho}(\rho, q, u)\right\rangle=0, \forall \psi
$$

where the scalar product $\langle *, *\rangle_{\rho}$ is defined by

$$
\begin{gathered}
\left\langle\psi, C_{\rho}\right\rangle_{\rho}=\sum_{j=1}^{J}\left\{\int_{0}^{1} \psi_{j}(x, T) \rho_{j}(x, T)-\psi_{j}(x, 0) \rho_{j}^{I}(x) d x\right. \\
\left.+\int_{0}^{T} v_{j} \psi_{j}(1, t) \rho_{j}(1, t)-\psi_{j}(0, t) f_{j}(q, u) d t-\int_{0}^{T} d t \int_{0}^{1} d x\left(\partial_{t} \psi_{j} \rho_{j}+v_{j} \partial_{x} \psi_{j} \rho_{j}\right)\right\}
\end{gathered}
$$


Similarly, we define the constraints $C_{q}(\rho, q, u)=0$ in (15) weakly by

$$
C_{q}(\rho, q, u)=0 \Longleftrightarrow\left\langle\phi, C_{q}(\rho, q, u)\right\rangle_{q}=0, \forall \phi,
$$

where the scalar product $\langle *, *\rangle_{q}$ is defined by

$$
\left\langle\phi, C_{q}(\rho, q, u)\right\rangle_{q}=
$$

$\sum_{j=1}^{J}\left\{\phi_{j}(T) q_{j}(T)-\phi_{j}(0) q_{j}^{I}+\int_{0}^{T}-\partial_{t} \phi_{j} q_{j}+\phi_{j}\left[f_{j}(q, u)-\sum_{k=1}^{J} A_{j k}(u) v_{k} \rho_{k}(1, t)-\lambda_{j}\right] d t\right\}$

Thus, we have $J$ test functions, or Lagrange multipliers, $\psi_{j}(x, t)$ for the constraints $C_{\rho}$ in (14), and $J$ Lagrange multipliers $\phi_{j}(t)$ for the constraints $C_{q}$ in (15). Optimizing the unconstrained functional in (18) now corresponds to setting all the Frechet derivatives w.r.t $\rho, q, u, \psi, \phi$ equal to zero. This gives in weak form

(a) $D_{\rho} G(\rho, q, u) \delta \rho+\left\langle\psi, D_{\rho} C_{\rho}(\rho, q, u) \delta \rho\right\rangle_{\rho}+\left\langle\phi, D_{\rho} C_{q}(\rho, q, u) \delta \rho\right\rangle_{q}=0 \forall \delta \rho$,

(b) $D_{q} G(\rho, q, u) \delta q+\left\langle\psi, D_{q} C_{\rho}(\rho, q, u) \delta q\right\rangle_{\rho}+\left\langle\phi, D_{q} C_{q}(\rho, q, u) \delta q\right\rangle_{q}=0 \forall \delta q$,

(c) $D_{u} G(\rho, q, u) \delta u+\left\langle\psi, D_{u} C_{\rho}(\rho, q, u) \delta u\right\rangle_{\rho}+\left\langle\phi, D_{u} C_{\rho}(\rho, q, u) \delta u\right\rangle_{\rho}=0 \forall \delta u$,

$$
\text { (d) } C_{\rho}(\rho, q, u)=0, \quad(e) C_{q}(\rho, q, u)=0
$$

\subsection{The minimizer equations}

The equations (21), determining the optimizer of the Lagrangian, the unconstrained functional in (18), can be re-written in their strong from again, leading to the solution of a set of differential algebraic equations for the Lagrange multipliers $\psi_{j}, \phi_{j}$ and the controls $u$. To do so, we note that the functional $G(\rho, q, u)$ maps its arguments into the real numbers. Therefore, its functional derivatives $D_{\rho} G, D_{q} G, D_{u} G$ are linear operators into $\mathbb{R}$, and can be expressed in terms of integral kernels. We denote these kernels by $K_{j}^{\rho}(x, t), K_{j}^{q}(t)$ and $K_{n}^{u}(t)$, satisfying

$$
\text { (a) } D_{\rho} G(\rho, q, u) \delta \rho=\sum_{j=1}^{J} \int_{0}^{T} d t \int_{0}^{1} d x K_{j}^{\rho}(\rho, q, u) \delta \rho_{j}
$$




$$
\begin{aligned}
& \text { (b) } D_{q} G(\rho, q, u) \delta q=\sum_{j=1}^{J} \int_{0}^{T} K_{j}^{q}(\rho, q, u) \delta q_{j} d t \\
& \text { (c) } D_{u} G(\rho, q, u) \delta u=\sum_{n=1}^{N} \int_{0}^{T} K_{n}^{u}(\rho, q, u) \delta u_{n} d t
\end{aligned}
$$

\section{The equation for $\psi$ :}

To compute the strong form of (21)(a) we differentiate the unconstrained functional (18) w.r.t. $\rho$, using (19)-(20) and obtain

$$
\begin{aligned}
& \sum_{j}\left\{\int_{0}^{T} d t \int_{0}^{1} d x K_{j}^{\rho}(\rho, q, u) \delta \rho_{j}+\int_{0}^{1} \psi_{j} \delta \rho_{j}(x, T) d x+\int_{0}^{T} v_{j} \psi_{j}(1, t) \delta \rho_{j} \psi_{j}(1, t) d t\right. \\
& \left.-\int_{0}^{T} d t \int_{0}^{1} d x\left[\partial_{t} \psi_{j}+v_{j} \partial_{x} \psi_{j}\right] \delta \rho_{j}-\sum_{k} \int_{0}^{T} \phi_{j} A_{j k}(u) v_{k} \delta \rho_{k}(1, t) d t\right\}=0 \forall \delta \rho
\end{aligned}
$$

This is clearly the weak formulation of the terminal - boundary value problem

$$
\text { (a) } \partial_{t} \psi_{j}+v_{j} \partial_{x} \psi_{j}=K_{j}^{\rho}(\rho, q, u)
$$

$$
\text { (b) } \psi_{j}(x, T)=0, \quad \psi_{j} v_{j}(1, t)=\sum_{k=1}^{J} \phi_{k}(t) A_{k j}(u) v_{j}, \quad j=1, . ., J
$$

\section{The equation for $\phi$ :}

Repeating the above exercise, computing the strong form of (21)(b) by differentiating the unconstrained functional (18) w.r.t. $q$ and using (19)-(20), we obtain

$$
\begin{gathered}
\sum_{j}\left\{\int_{0}^{1} d x \int_{0}^{T} d t K_{j}^{q}(\rho, q, u) \delta q_{j}-\int_{0}^{T} \psi_{j}(0, t) \sum_{k} \partial_{q_{k}} f_{j}(q, u) \delta q_{k} d t+\phi_{j}(T) \delta q_{j}(T)\right. \\
+\int_{0}^{T}\left[-\partial_{t} \phi_{j} \delta q_{j}(t)+\phi_{j} \sum_{k} \partial_{q_{k}} f_{j}(q, u)\right] \delta q_{k} d t \forall \delta q
\end{gathered}
$$

This is clearly the weak formulation of the terminal - value problem

$$
\text { (a) } \partial_{t} \phi_{j}=\sum_{k=1}^{J}\left[\phi_{k}-\psi_{k}(0, t)\right] \partial_{q_{j}} f_{k}(q, u)+\int_{0}^{1} d x \quad K_{j}^{q}(\rho, q, u)
$$


(b) $\phi_{j}(T)=0, \quad j=1, . ., J$

\section{The equation for $u$ :}

We have to do this one more time, computing the strong form of (21)(c) by differentiating the unconstrained functional (18) w.r.t. $u$ and using (19)-(20), we obtain

$$
\begin{gathered}
K_{n}^{u}(q, u, t)+\sum_{j}\left[\phi_{j}-\psi_{j}(0, t)\right] \partial_{u_{n}} f_{j}(q, u)-\sum_{j} \sum_{k} \phi_{j} \partial_{u_{n}} A_{j k}(u) v_{k} \rho_{k}(1, t)=0 \\
n=1, . ., N
\end{gathered}
$$

\subsection{Steepest descent and the Gauss - Seidel iteration}

Solving the Lagrangian equations (21), using the form (23), (24), (25) of the individual equations looks like a quite formidable computational task, since we have doubled the number of simulation variables and have to deal with a coupled forward-in-time and backward-in-time problem. Moreover, the problem will in general have multiple solutions, for the simple reason that there will be regions in the space - time domain where the controls do not affect the solution. This implies that there are in general many optimal controls. These problems can be avoided by computing the optimum via a steepest descent method and by solving (21) via a block Gauss - Seidel approach. We note that equations $(21)(\mathrm{a})(\mathrm{b})(\mathrm{d})(\mathrm{e})$ are actually easily solved for a given control $u$. Solving $(21)(\mathrm{d})(\mathrm{e})$ corresponds to just a forward simulation of the network, i.e. solving (14)-(15) for a given $u$. Once $\rho$ and $q$ are obtained this way, solving (21)(a)(b), or (23), (24) respectively, corresponds to solving a problem of essentially the same structure and complexity backwards, i.e. to solving the dual network problem. This leaves only equation $(21)(\mathrm{c})$, or (25) respectively, to deal with. We use the following idea. We formally denote the solution operator of the forward network problem (14)-(15) for a given $u$ by $(\rho, q)=F(u)$. Solving the constrained optimization problem now formally corresponds to minimizing the unconstrained functional

$$
g(u)=G(F(u), u)
$$

To solve this problem by a steepest descent method, we would have to compute the gradient direction of $g$ for a given iterate $u$, i.e. We would have to 
compute $D_{u} g$ given by

$$
D_{u} g \delta u=D_{\rho q} G(F, u) D_{u} F(u) \delta u+D_{u} G(F, u) \delta u
$$

Note that $g$ and $G$ are functionals into the real numbers, and so their derivatives can be identified with functions. So the above reads

$$
\begin{gathered}
\sum_{n=1}^{N} \int_{0}^{T} D_{u_{n}} g(t) \delta u_{n}(t) d t=\sum_{n=1}^{N} \int_{0}^{T}\left[D_{\rho q} G(F, u) D_{u} F(u)+D_{u} G(F, u)\right]_{n}(t) \delta u_{n}(t) d t, \forall \delta u \\
D_{u_{n}} g(t)=\left[D_{\rho q} G(F, u) D_{u} F(u)+D_{u} G(F, u)\right]_{n}(t)
\end{gathered}
$$

Of course $F(u)$ and its derivative is not explicitly available. However it can be shown that for a given control $u$ the term $\left[D_{\rho q} G(F, u) D_{u} F(u)+\right.$ $\left.D_{u} G(F, u)\right]_{n}(t)$ is the residual in equation (21)(c) when solving (21)(a,b,d,e). This leads to the following algorithm

- For a given control $u(t)$

- Solve the network problem $(21)(\mathrm{d}, \mathrm{e})$, i.e. solve equations (14)-(15)
(a) $\partial_{t} \rho_{j}+v_{j} \partial_{x} \rho_{j}=0$
(b) $v_{j} \rho_{j}(0, t)=f_{j}(q, u)$,
(c) $\rho_{j}(x, 0)=\rho_{j}^{I}(x)$,
(a) $\partial_{t} q_{j}+f_{j}(q, u)-\sum_{k=1}^{J} A_{j k}(u) v_{k} \rho_{k}(1, t)-\lambda_{j}=0$,
(b) $q_{j}(0)=q_{j}^{I}, \quad j=1, . ., J$.

- Now, given $\rho$ and $q$ solve the dual network problem (21)(a,b), i.e. solve (23) and (24) backward in time:

$$
\text { (a) } \partial_{t} \psi_{j}+v_{j} \partial_{x} \psi_{j}=K_{j}^{\rho}(\rho, q, u),
$$

$$
\begin{gathered}
\text { (b) } \psi_{j}(x, T)=0, \quad \psi_{j} v_{j}(1, t)=\sum_{k=1}^{J} \phi_{k}(t) A_{k j}(u) v_{j}, \quad j=1, . ., J \\
\text { (a) } \partial_{t} \phi_{j}=\sum_{k=1}^{J}\left[\phi_{k}-\psi_{k}(0, t)\right] \partial_{q_{j}} f_{k}(q, u)+\int_{0}^{1} d x \quad K_{j}^{q}(\rho, q, u)
\end{gathered}
$$

(b) $\phi_{j}(T)=0, \quad j=1, . ., J$ 
- Compute the gradient direction $D_{u_{n}} g$ from the residual in $(21)(\mathrm{c})$, according to $(25)$

$$
\begin{gathered}
D_{u_{n}} g(u)=K_{n}^{u}(q, u, t)+\sum_{j}\left[\phi_{j}-\psi_{j}(0, t)\right] \partial_{u_{n}} f_{j}(q, u)-\sum_{j} \sum_{k} \phi_{j} \partial_{u_{n}} A_{j k}(u) v_{k} \rho_{k}(1, t), \\
n=1, . ., N
\end{gathered}
$$

- Advance $u$ in the direction of $-D_{u} g$ using the standard steepest descent approach.

\section{Discrete controls}

In this section we discuss the case $u_{i}(t) \in\{0,1\}$ which implies that at each time step the processor can decide between a 'PUSH' or 'PULL' policy, see section 2.4. The presented approach can be seen as computational alternative to the adjoint calculus presented before exploiting the discrete nature of the underlying decision. The idea consists in reformulating the network optimization problem (17) as mixed-integer programming problem by suitable discretization of the dynamics governed by partial and ordinary differential equation. These approach has been successfully applied in other applications, e.g., traffic flow [11], or for routing problems in supply chain models [12]. To distinguish the present approach from existing work and for ease of presentation, we introduce the following assumptions on the policy network model given by equations (14), (15) and (9).

A1 We do not consider optimal routing, i.e., the connectivity matrix $A$ in equation (15) is independent of the control $u$.

A2 We consider production lines were each part has to go at most two times through the same processor. The example of section 2.4 is of this type and the possible policies at a single processor $i$ at time $t$ are therefore either 'PUSH' (say corresponding to $u_{i}(t)=0$ ) or 'PULL', i.e., $u_{i}(t)=1$.

A3 The cost functional $G(\rho, q, u)$ is linear and of integral type, e.g.,

$$
G(\rho, q, u)=\sum_{j=1}^{J} \int_{0}^{T}\left(\int_{0}^{1} c^{\rho} \rho_{j}(x, t) d x\right)+c^{q} q_{j} d t
$$


Remark 4.1 The assumption (A1) can easily be removed, see [14]. Further, assumption (A3) can be removed and nonlinear cost functionals $G(\rho, q, u)$ can be included when treated as presented e.g. in [22].

The details of the discretization are as follows: Let $\Delta x=1$ be the length of each processor. Then, $\Delta t=\min \left\{\epsilon, 1 / v_{j}: j=1, \ldots, J\right\}$ is temporal width of the time grid satisfying the CFL condition. We denote by $\rho_{j, l}^{1}, \rho_{j, l}^{b}$ and $q_{j, l}$ an approximation to the density $\rho_{j}(0, l \Delta t), \rho_{j}(1, l \Delta t)$ and $q_{j}(l \Delta t)$, respectively. The two-point Upwind discretization of (14) reads

$$
\rho_{j, l+1}^{b}=\rho_{j, l}^{b}+\frac{\Delta t}{\Delta x} v_{j}\left(\rho_{j, l}^{a}-\rho_{j, l}^{b}\right)
$$

and the explicit Euler discretization of (15) for $\lambda_{j, l}=\lambda_{j}(l \Delta t)$ is given by

$$
q_{j}^{l+1}=q_{j}^{l}+\Delta t\left(\sum_{k=1}^{J} A_{j k} v_{k} \rho_{k, l}^{b}-v_{j} \rho_{j, l}^{a}\right)+\lambda_{j, l} .
$$

The initial data is $q_{j}^{0}=q_{j}^{I}, \rho_{j, 0}^{a}=\rho_{j}^{I}(0)$ and $\rho_{j, 0}^{b}=\rho_{j}^{I}(1)$, respectively. The cost functional is discretized by trapezoidal rule in space and time. It remains to discuss the proper discretization of equation (9) with $S(j)=2$ due to assumption (A2) but depending on $u_{i}(t), i=1, \ldots, N$ : at a fixed processor $j$ with virtual processors $j 1$ and $j 2$ and control $u_{i}$, equation (9) reads

$$
\begin{array}{r}
v_{j} \rho_{j 1}(0, t)=f_{j 1}=\min \left\{c_{j}-u_{i}(t) f_{j 2}, \frac{v_{j} q_{j 1}(t)}{\epsilon}\right\}, \\
v_{j} \rho_{j 2}(0, t)=f_{j 2}=\min \left\{c_{j}-\left(1-u_{i}(t)\right) f_{j 1}, \frac{v_{j} q_{j 2}(t)}{\epsilon}\right\}
\end{array}
$$

Obviously, for $u_{i}=0$ we obtain the 'PUSH' policy, i.e., processor $j 1$ has higher priority than $j 2$ and vice versa the 'PULL' policy for $u_{i}=1$ (compare with section 5). Using discrete controls $u_{i, l} \in\{0,1\}$, additional binary variables $\xi_{j s, l}$ and real variables $\mathbf{f}_{j s, l}^{0}$ and $\mathbf{f}_{j s, l}^{1}$ for $s=1,2$, we reformulate (29) and (30) by 


$$
\begin{aligned}
& \mathbf{f}_{j s, l}^{0}+\mathbf{f}_{j s, l}^{1}=\quad v_{j} \rho_{j s, l}^{a}, \\
& 0 \leq \mathbf{f}_{j s, l}^{1} \leq u_{i, l} M, \quad 0 \leq \mathbf{f}_{j s, l}^{0} \leq\left(1-u_{i, l}\right) M, \\
& c_{j} \xi_{j s, l} \leq v_{j} \rho_{j s, l}^{a}+\mathbf{f}_{j(3-s), l}^{(2-s)} \leq c_{j} \\
& v_{j} \frac{q_{j s, l}}{\epsilon}-M \xi_{j s, l} \leq \quad v_{j s} \rho_{j s, l}^{a} \leq v_{j} \frac{q_{j s, l}}{\epsilon} \\
& c_{j} \xi_{j s, l} \leq v_{j} \frac{q_{j s, l}}{\epsilon}+\mathbf{f}_{j(3-s), l}^{(2-s)} \leq c_{j}\left(1-\xi_{j s, l}\right)+M \xi_{j s, l}
\end{aligned}
$$

for $s=1,2$ and $M$ a sufficiently large constant. To obtain the equivalence of (31) - (35) and (29)-(30) several cases have to be considered. With the other cases being similar we assume a 'PUSH' policy at time $l \Delta t$ and $v_{j} q_{j 1, l} \leq \epsilon c_{j}$. Then, (31) and (32) imply $v_{j} \rho_{j s, l}^{a}=\mathbf{f}_{j s, l}^{0}$ and $\mathbf{f}_{j s, l}^{1}=0$. Further, (35) implies $\xi_{j 1, l}=0$. Due to (34),

$$
v_{j} \rho_{j 1, l}^{a}=v_{j} \frac{q_{j 1, l}}{\epsilon}=\mathbf{f}_{j 1, l}^{0}
$$

and then depending on whether $c_{j}-\left(v_{j} \frac{q_{j 2, l}}{\epsilon}+\mathbf{f}_{j 1, l}^{0}\right)$ is positive or negative we obtain either $\xi_{j 2, l}=1$ or $\xi_{j 2, l}=0$, respectively, and therefore

$$
v_{j} \rho_{j 2, l}^{a}=\min \left\{c_{j}-\mathbf{f}_{j 1, l}^{0}, \quad v_{j} \frac{q_{j 2, l}}{\epsilon}\right\} .
$$

Summarizing, the discretized optimal control problem reads

$\min _{\rho_{j, l}^{a}, \rho_{j, l}^{b}, q_{j, l}, u_{i, l}, \xi_{j, l}, \mathbf{f}_{j, l}^{0}, \mathbf{f}_{j, l}^{1}} \Delta t \sum_{j, l}\left(\frac{\Delta x}{2} c_{j}^{\rho}\left(\rho_{j, l}^{a}+\rho_{j, l}^{b}\right)+c_{j}^{q} q_{j, l}\right)$ sbj. to $(27,28,31-35)$

for $u_{i, l}$ and $\xi_{j, l}$ binary and $\lambda_{i, l}$ a given inflow and $c_{j}^{\rho}$ and $c_{j}^{q}$ given costs.

\section{$5 \quad$ Numerical experiments}

First, we consider the example presented in section 2.4 and illustrate the effect of different policies on the production process. We assume a maximal capacity of each processor of $c_{j}=1$ and a processing velocity of $v_{j}=1$. We prescribe a piecewise linear inflow profile $\lambda_{i}(t)$ and compare the outflow profile $M 5$ and the trash $M 6$ over a time horizon of $T=120$. In figure 2 we observe that in the 'PUSH' policy at $T=65$ no more parts arrive in the 

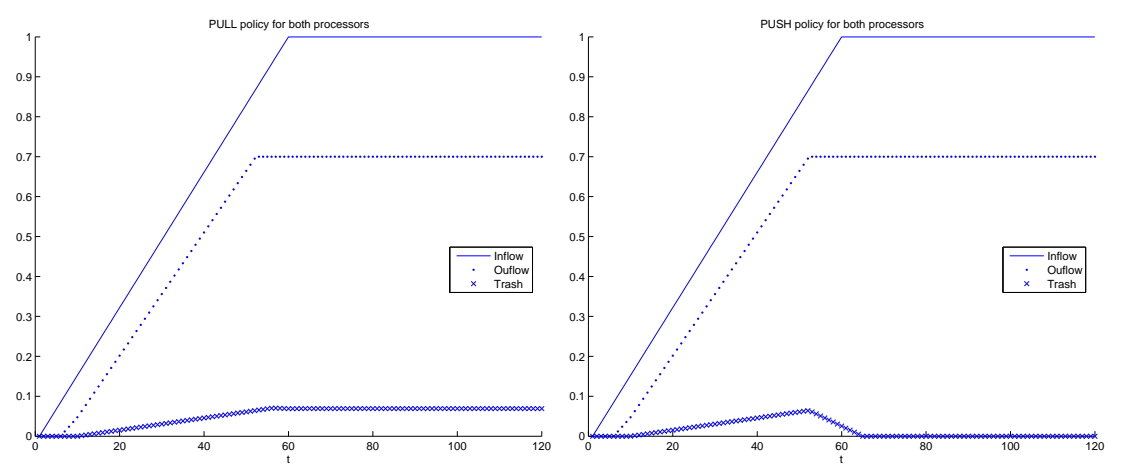

Figure 2: Time evolution of influx (solid), trash (dotted) and output processor $(\times)$ for 'PULL' (left panel) and 'PUSH' (right panel) policy for production line $(1)$.

trash, since the first processor uses its full capacity $\left(c_{j}=1\right)$ to process the incoming flow.

The second example is a re-entrant line introduced by $\mathrm{Lu}$ and Kumar $[17,10]$ depicted in figure 3 . A part has to pass processor one and then twice processor two, before again passing through processor one and exiting. Each processor can decide independently which pass is given a higher priority. As in the example of section 2.4 we say the processors works in 'PUSH' policy, if its first pass has higher priority.

We assume a maximal capacity $c_{1}=1$ and $c_{2}=1.25$ and velocity $v_{j}=1$. We consider an optimization horizon of $T=30$ and a boxed shaped inflow profile with a maximum of 0.8 for $10 \leq t \leq 17$ and 0.3 else which is also the initial value for all processors. The inflow profile is chosen such that the timeaverage inflow (0.43) is below the effective capacity of both processors. In figure 3 we present outflow profiles and the total outflow for different (timeindependent) policies, e.g., the 'PULL'-'PUSH' policy corresponds to the production line in $\rightarrow M_{4} \rightarrow M_{2} \rightarrow M_{3} \rightarrow M_{1} \rightarrow$ out. Despite the fact that the average workload is less than the effective capacities, none of the timeindependent policies let all incoming parts pass through the network due to successive blocking. To obtain an optimal switching policy for each processor we now solve the network optimization problem with a cost functional given by

$$
G(\rho, q, u)=\int_{0}^{T} \rho_{4}(1, t) d t
$$


First, we consider the case of discrete controls as outlined in section 4 . The mixed-integer problem (38) is solved using ILOG CPLEX V10 [15]. The optimal switching policies and the corresponding outflow profile for this case is depicted in figure 4. We observe that due to the dynamic policy switching all parts can be processed through the network. Furthermore, the optimal switching policy of the second processors is similar to the policy of its predecessor but delayed by one time-step corresponding to the imposed processing velocity of $v_{j}=1$. Second, we implement a steepest descent method for the continuous convex combination of PUSH and PULL policies as outlined in section 2.5 and 3.3. To compare the results with the mixed-integer approach we also use a two-point Upwind and an explicit Euler discretization for the forward equation (10) and (11) as well as for the adjoint equation (23) and (24). The gradient of the reduced cost functional is then evaluated according to (25). The gradient is then used in a steepest descent type method with Armijo step-size rule, see e.g. [16, 23]. To ensure that the control is bounded $0 \leq u_{i}(t) \leq 1$ we complement our steepest descent with a projection on $[0,1]$, see e.g., [20]. We stop the algorithm if the norm of the reduced gradient is less than $10^{-5}$. All other parameters are as in the mixed-integer example. The results are shown in figure 5. In particular, we observe that there is no uniqueness to the optimal policy in this example. In the continuous case one solution is depicted in the left part of figure 5 which is a convex combination of PULL and PUSH properties. Note that, in general, the optimum achieved by a continuous policy has to be at least as good as the one achieved by the discrete policy, since we optimize over a wider range of possible policy choices.

\section{Conclusions}

We introduced a supply chain model for networks with the additional possibility to give certain parts priorities in the processing order. We discussed some examples and formulated an optimization problem to determine an optimal policies and optimal routing descisions subject to capacity, inflow and geometry data of the supply chain. Depending on whether the policy is discrete (e.g. PUSH or PULL) or continuous (e.g. convex combination of PUSH and PULL) we formulated a mixed-integer programming problem and derived an adjoint calculus for characterization and computation of the optimal policy. Both approaches are illustrated on a sample network of a re- 

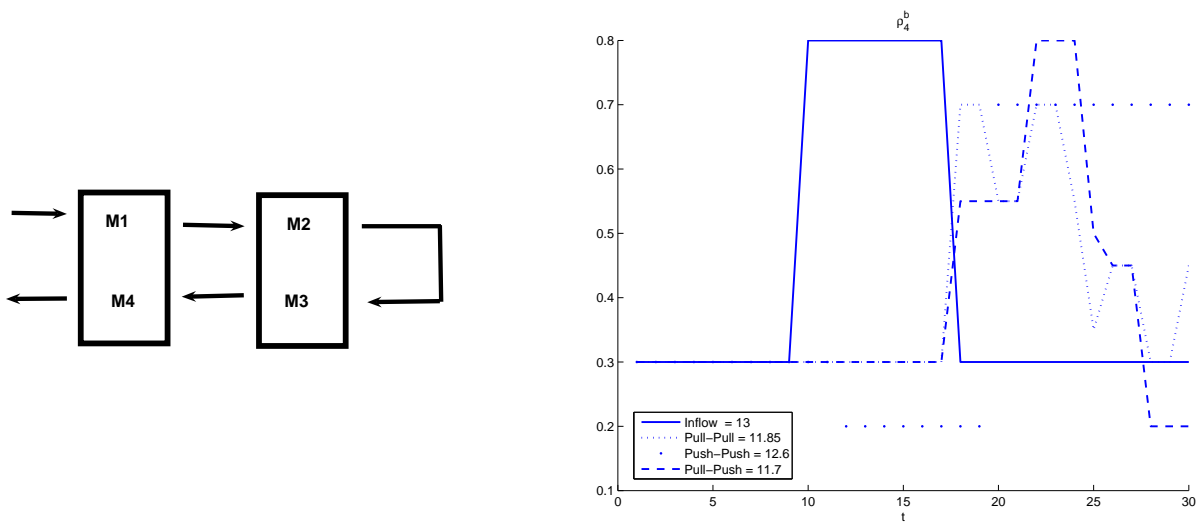

Figure 3: Re - entrant line with two processors and inflow, outflow profile and total outflow for different policies.

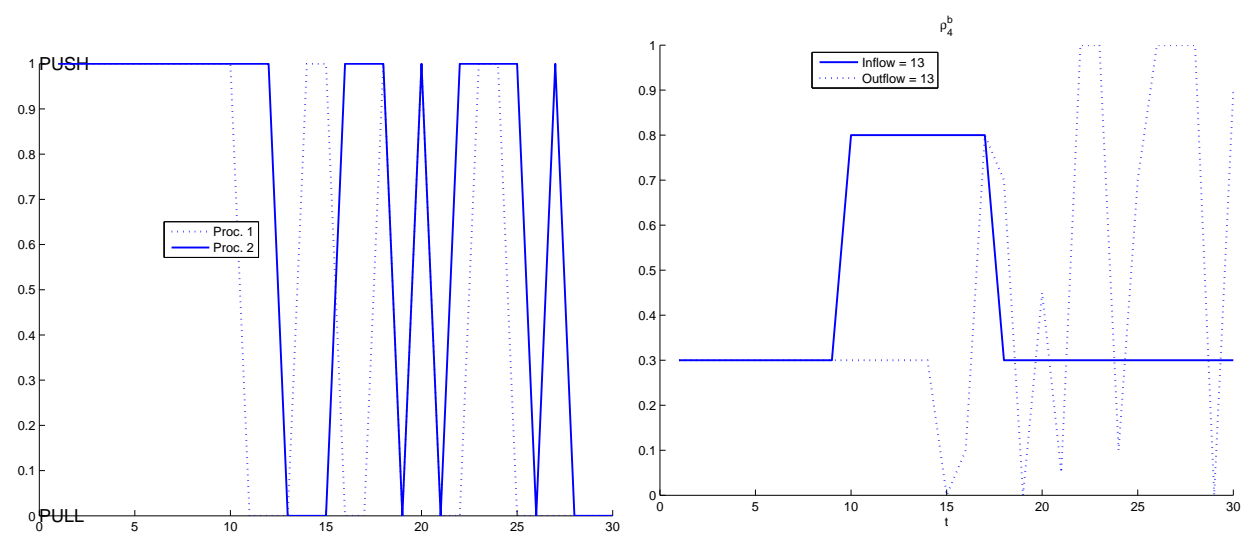

Figure 4: Optimal discrete switching policies (left panel) and corresponding outflow profile and total outflow (right panel) for the Lu-Kumar network.

entrant factory with two processing stages and transient inflow profile. We numerically solve for the optimal policies and show that other, naive choices of processing policies do not perform well on this example.

\section{Acknowledgements}

M. Herty wants to thank the Arizona State University for their hospitality. 

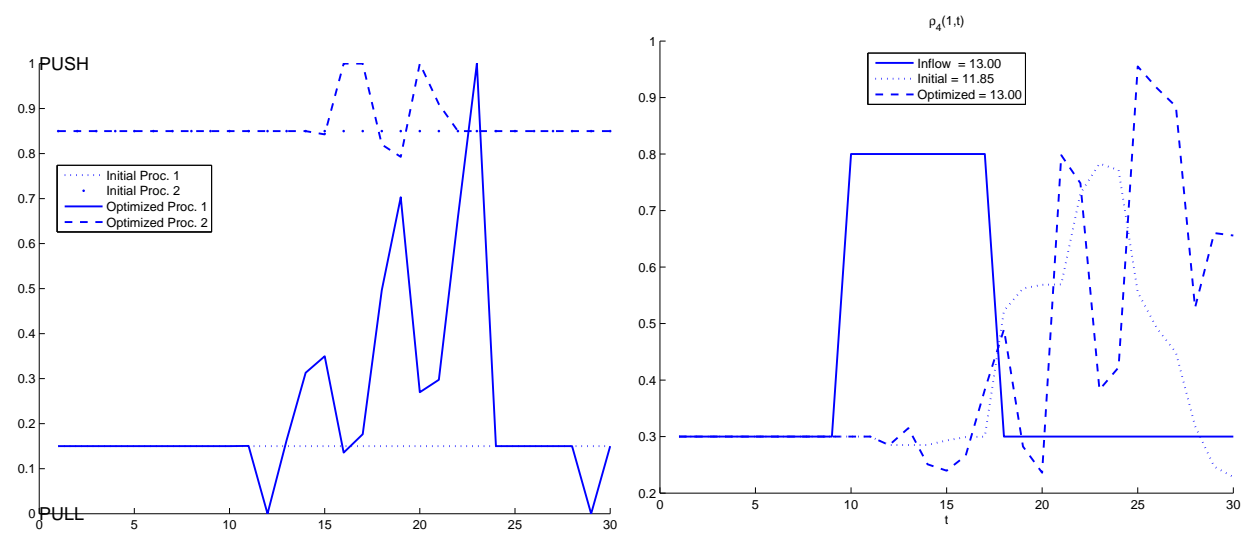

Figure 5: Continuous policies (left panel) and corresponding outflow profile and total outflow (right panel) for Lu-Kumar network.

\section{References}

[1] E.J. Anderson, A new continuous model for job scheduling, International J Systems Science 12: 1469-1475, 1981.

[2] D. Armbruster, C. De Beer, M. Freitag, T. Jagalski, C. Ringhofer, Autonomous Control of Production Networks using a Pheromone Approach, PHYSICA A, 363(1):104-114, 2006.

[3] D. Armbruster, D. Marthaler, C. Ringhofer, A mesoscopic approach to the simulation of semiconductor supply chains, Proceedings of the International Conference on Modeling and Analysis of Semiconductor Manufacturing (MASM2002), G. Mackulak et al, eds., 365 369, 2002.

[4] D. Armbruster, D. Marthaler, C. Ringhofer, Kinetic and Fluid Model Hierarchies for Supply Chains, SIAM J. on Mutiscale Modeling and Simulation, 2:43-61, 2004.

[5] D. Armbruster, C. Ringhofer, Thermalized kinetic and fluid models for re-entrant supply chains, SIAM J. Multiscale Modeling and Simulation, 3:782-800, 2005. 
[6] D. Armbruster, P. Degond, C. Ringhofer, A Model for the Dynamics of large Queuing Networks and Supply Chains SIAM J. Appl. Math. 66: 896-920 , 2006.

[7] J. Banks, J. Carson II, B. Nelson, Discrete event system simulation, Prentice Hall , 1999.

[8] R.E. Bixby, M. Fenelon, Z. Gu, E. Rothberg and R. WunDERLING, MIP: Theory and Practice - Closing the Gap, System Modelling and Optimization, 19 - 50, 1999.

[9] C. Daganzo, A Theory of supply chains, Springer-Verlag, Heidelberg, Germany, 2003.

[10] J. G. DAI AND G. WeISS, Stability and instability of fluid models for re-entrant lines, Mathematics of Operations Research, 21:115-135, 1996.

[11] A. Fügenschuh, M. Herty, A. Klar and A. Martin, Combinatorial and Continuous Models for the Optimization of Traffic Flows on Networks, SIAM J. Optimization 16(4):1155 - 1176, 2006.

[12] A. Fügenschuh, S. Göttlich, M. Herty, A. Klar and A. MARTin, A mixed-integer programming approach for the optimization of continuous models in supply chain management, preprint 2006.

[13] S. Göttlich, M. Herty and A. Klar, Network models for supply chains, Comm. Math. Sci., Vol.3(4):545-559, 2005.

[14] S. Götthich, M. Herty, C. Kirchner, A. Klar, Optimal control for continuous supply network models, Proceedings of the NHM Launching Meeting, 2006

[15] Ilog CPleX Division, 889 Alder Avenue, Suite 200, InCline Village, NV 89451, USA, Information available at URL http://www.cplex.com.

[16] C. KeLleY, Iterative methods for optimization, SIAM Frontiers in Applied Mathematics, 1999. 
[17] P. R. Kumar And S. H. Lu, Distributive scheduling based on due dates and buffer priorities, IEEE Transaction on Automatic Control, 36:140-1416, 1991.

[18] M. Lighthill, J. Whitham, On kinematic waves, I: Flow movement in long rivers, II: A theory of traffic flow on long crowded roads, Proc. Royal. Soc., A 229: 281-345, 1955.

[19] D. Marthaler, D. Armbruster, C. Ringhofer, K.Kempf, T.C. Jo, A continuum model for a re-entrant factory, Operations Research 54 (5):933-951, 2006.

[20] J. J. More And G. Toraldo, Algorithms for bound constraint quadratic programming problems, Num. Math., 55:377, 1989.

[21] G.F. Newell, A simplified theory of kinematic waves in highway traffic, Transportation Research B 27:281-313, 1993.

[22] G. Nemhauser And L.A. Wolsey, Integer and Combinatorial Optimization, Wiley-Interscience, 1999.

[23] P. Spellucci, Numerische Verfahren der nichtlinearen Optimierung, Birkhäuser Verlag, Basel, Boston, Berlin, 1993. 